\title{
Telerobotic exploration and development of the Moon
}

\author{
B L Cooper ${ }^{1, *}$, B Sharpe ${ }^{2}, \mathrm{D} \mathrm{SchrunK}^{3}$ and M Thangavelu ${ }^{4}$ \\ ${ }^{1}$ Oceaneering Space Systems, Houston, Texas, USA. \\ ${ }^{2}$ Independent Lunar Development Planner, St. Louis, MO, USA. \\ ${ }^{3}$ Quality of Laws Institute, Poway, CA, USA. \\ ${ }^{4}$ Space Exploration Architectures Concept Synthesis Studio, Division of Astronautics and \\ Space Technology and School of Architecture, University of Southern California, USA. \\ *e-mail: bcooper138@houston.rr.com
}

\begin{abstract}
There has been a debate for the last thirty years about the relative merits of human versus robotic systems and we argue here that both are essential components for successful lunar exploration and development. We examine the role of robots in the next phases of exploration and human development of the Moon. The historical use of robots and humans in exploration is discussed, including Apollo-era exploration, International Space Station, and deep-water petroleum exploration. The technological challenges of lunar operations are addressed in the context of how robotic systems can be designed for robust and flexible operations. System design recommendations are given based on the lessons learned from terrestrial and space robotics.
\end{abstract}

\section{Introduction}

The Moon is becoming the principal focus of the space exploration and development efforts of national space agencies. Following the lunar missions that are currently in development by many different countries, an international lunar base is expected to be established in the next several years. The first elements of the lunar base will be assembled with robotic precursor missions, and human missions will follow, perhaps within a decade.

There has been a debate for the last thirty years about the relative merits of human versus robotic exploration. Proponents of robotic exploration point out that it's much less expensive to send robots into space than it is to send humans, and that robots, as extensions of our senses, are our 'virtual presence' in the solar system. They argue that robots can do anything that humans can do, and they can do it cheaper. On the other hand, people who worked on the Apollo missions argue that humans have unique capabilities for on the spot thinking, observing, and reasoning. They list examples of how humans can fix things that break, improvise when plans change, and take advantage of unexpected opportunities. Most of this discussion has focused on the 'either/or' line of reasoning.

We argue that the 'both/and' approach is more useful, and we examine the role of robots in the next phases of exploration and human development of the Moon.

\subsection{Apollo experience}

Telerobotic devices were successfully operated on the lunar surface in the 1960s and 1970s during the Luna, Surveyor, and Apollo programs. Spacecraft instruments were commanded mainly in real-time by operators on Earth, and receipt confirmed in control areas via the available tools - strip charts, printouts and still and video images of various resolutions.

Controlling lunar surface devices was relatively cheap and simple, compared to other space operations. The Moon is a cooperative target for earthbased antennas in terms of its position in the sky and near-unchanging distance; and pointing

Keywords. Telerobotics; lunar exploration development.

J. Earth Syst. Sci. 114, No. 6, December 2005, pp. 815-822

(C) Printed in India. 
antennas on the Moon's surface back toward Earth is also relatively simple. The physical environment is predictable, and in itself contains nothing requiring fast or complicated reactions. Moreover, there is little to cause hardware deterioration, the main design considerations being long-term effects of radiation (including solar flares), meteorites, temperature extremes, and dust. A new generation of devices could be made to last indefinitely, provided sufficient safeguards were designed into the hardware and operating procedures.

With today's available and emerging technologies, earth-bound operators and audiences could have a far greater sense of participation. They could 'work on the Moon' every day, and be home for dinner.

\subsection{International space station experience}

Currently, the number of space walks needed for assembly and maintenance of ISS is so large that there is little time left over for science. Designers did not fully appreciate that the complexity and newness of the ISS would translate into unexpected maintenance. Now, NASA is exploring ways to use robots that are operated either by the crew onboard the station, or by ground control personnel, for maintenance - so that fewer space walks will be needed, and more time can be used for scientific research.

\subsection{Deep-ocean experience}

In the last 20 years, we have learned that the optimum method of exploring the submarine environment involves both humans and robots. However, we do not send robots out to do this work and keep all the humans in the office. The humans go out on the ship or platform so they can be close to the action. That way, they can fix things that the robots cannot fix, react to surprises, and have as close a viewpoint as possible to what is going on.

In deep-sea drilling operations, divers are frequently accompanied by remotely-operated vehicles, whose principal job is to fetch and carry tools, and provide a view of the operations for the people at the surface. Note that the petroleum industry is classified as a 'for profit' institution - they aggressively look for the most efficient and economical way to do business. The fact that they use robots is strong evidence for the usefulness of this technology.

\subsection{Latency}

Radio signals take about three seconds to make a round-trip from the Earth to the Moon and back.
This amount of time lag, called latency, is the time it takes an earth-bound operator to experience the results of an action. In highly dynamic situations such as driving a vehicle at appreciable speed, latency would be a problem, and an operator could quickly find himself in a disorienting situation, potentially damaging to the equipment or work. There are two ways of handling the latency problem: first, proceed slowly; and second, use automated subroutines (as discussed in section $2.1 \mathrm{~b})$.

\section{Technological challenges}

\section{1a Go slowly}

Going slowly means designing operations so that the situation does not get ahead of the operator's ability to control it. On the Moon there is nothing in the natural environment that will require fast response to an unanticipated event. Quick reactions would only be required in contingency situations if something unexpected happened, and keeping a slow pace will reduce the chances of those occurrences.

Going slowly has other benefits. In the case of mobility devices, less lunar dust (a nemesis of thermal control) will be disturbed and redeposited where it is not wanted. Going slowly requires less bandwidth for control and uses less energy for mobility - and it lessens the harmful side effects from dissipating the heat that would otherwise be caused by expending energy for speed. In the Moon's vacuum, the only practical way to get rid of excess heat is by radiation. The only downside to working slowly may be a human one, i.e., impatience with a scheme of things that does not match the way things would be done on Earth. Slow-paced lunar operations might not only be found boring to watch, but there could be a tendency to think of them as inefficient, i.e., not getting things done 'fast enough'.

\section{$2.1 b$ Automated subroutines}

A second kind of lunar tele-operation will involve controlling devices that replicate the hand-eye coordination of a skilled technician. This is the kind of situation presently associated with surgical telemedicine. Two manipulators for hands, and two cameras for stereovision to an operator, along with some upper-body mobility, would be ideal. It will be useful to include processors and software in the lunar equipment that could control specific precise or repetitious operations, so that the role of the earth operator would be more supervisory. This method would be similar to the way many factory 
robot devices work on Earth. The control software would be fine-tuned and updated from time to time.

\subsection{Bandwidth requirements}

Sensory immersion in operator control stations requires high-bandwidth, high-resolution systems with stereo audio, imaging, and haptic (tactile) control (with or without feedback). Higher bandwidth does not eliminate the latency problem, but would provide more information that could be used for making better decisions. Device control as well as video and data could be stored as multi-channel records, which would later be used for analysis, training, and general distribution.

\subsection{Infrastructure requirements}

Initially, a facility to control lunar devices might resemble previous control centers. There would be command and display equipment for operators, as well as a network of computers, data lines, and transmitter-receiver stations at various places on Earth. Safeguards for both physical security from outside, and prevention of operator errors before they are made (via simulation and commandchecking) would be included.

Some work stations for operator control of devices (both 'mobility' and 'skilled technician' types) would be present, to provide a virtual lunar environment for some of the operators. This sense of being on the Moon could be distributed to the outside world via a virtual viewing-room environment for observers, with media feeds to the world at large. A control architecture would be chosen which would allow for eventual unlimited expansion of the numbers of devices on the lunar surface.

A long-term goal would be to distribute the tele-operation command capability to outside locations: corporations, universities, and individuals. Groups and individuals could then be assigned time slots to be on the Moon in a virtual sense, conducting activities of their own design for their own purposes.

\section{3a Lunar base requirements}

An essential requirement for tele-operations of robotic devices on the lunar surface is line-of-sight communications that enable earth-bound operators to have a virtual presence on the Moon, and long periods of sunlight to provide power for solar cells. Sharpe and Schrunk (2002) present a rationale for why the summit of Malapert Mountain, in the south polar region of the Moon, offers the optimum location for direct and continuous EarthMoon communications and long periods of continuous sunlight. Other sites that experience long periods of sunlight, such as Peary Crater at the North Pole and Shackleton Crater at the South Pole, are not continuously in line-of-sight with the Earth. For Earth-Moon communications, these polar sites would need to have a relay station placed on the lunar surface or in lunar orbit, which would add significantly to the cost and complexity of robotics operations. Thus, for the first robotic lander missions to the Moon, Malapert Mountain should be given consideration as the location of the first sunlight-dependent robotic base (Sharpe et al 2003).

\section{The robot associate study}

We performed a study recently ${ }^{1}$ to determine the most efficient and important ways to use robots in future lunar exploration. We found that there are specific, relatively easy, and very useful things that we can do with robots.

First we looked at tasks that would be best suited to robots in future space missions. Next, we prioritized the list of tasks, to understand which capabilities would offer the greatest return on investment. We wanted to find out which tasks would be easiest to develop into robotic tasks, while at the same time being the most beneficial for the mission.

\subsection{Study background}

Once we are on the surface of the Moon, you face a new set of challenges that are distinctly different from spacewalking on orbit. You have a partial gravity environment. Now, instead of just adding inertia, your space suit weighs something, and you have to deal with that weight as you move around. Also, you now have a sense of up and down. You can not change your orientation randomly to pick something up. If there is a rock on the ground that you want to look at, you must bend down to get it. You now have to worry about keeping your balance. We learned from Apollo that this can be very challenging in a 200-pound space suit. And because the gravity on the Moon is less than the gravity on Earth, you have problems that you would not encounter on Earth - such as the cables they used to deploy the Apollo surface experiments: there was not quite enough gravity to keep them flat, so they were a constant tripping hazard.

There are tasks which could be done robotically that would make spacewalks or planetary excursions more productive or easier. These 'Assistance'

\footnotetext{
${ }^{1}$ Funded by NASA Johnson Space Center's Automation, Robotics, and Simulation Division.
} 
tasks are fairly well understood because of our experience with robots in industry.

Exploration and development of the Moon will also involve robotic precursor missions that will arrive a couple of years ahead of the humans, prepare the base site, set up the modules, get the oxygen plant running, get the power plant running, and so on. Then the challenge is to keep everything running until the humans arrive. But if we want to send humans to the Moon, these robotic capabilities must be developed.

\subsection{Methodology}

A list of potential tasks for robotic automation or tele-operation was created. We then set up criteria to determine which tasks are both easy to roboticize and, at the same time, most beneficial. Each task was given a grade for aspects of difficulty and benefits, and the difficulty/benefit scores were tabulated. The goal was to find the tasks that are easy for robots to do (low scores on the difficulty scale) and tasks that are beneficial for robots to do (high scores on the benefit scale).

\section{$3.2 a$ Difficulty aspects}

\section{Technical risk}

For some tasks, such as inspection of a vehicle exterior, there are systems that are already spaceproven. The technical risk is thus low. For other systems, such as emplacing a lunar surface habitat, the hardware is all in the artwork phase. The technical risk here is comparatively high.

\section{Complexity of the task}

Some tasks are simple - they only require one or two steps; there may be only one or two objects to be manipulated, and the robot would not have to be very dexterous to do them. Obviously other tasks are more complicated, and simple tasks are easier to roboticize than complex ones.

\section{Robotic compatibility}

This is the general question of how well could robots (as we understand them) do this task? For example, inspecting debris shields for micrometeoroid damage is very compatible with robotics, whereas repairing those shields is not. On a planetary surface mission, observing a sample with a microscope is a robot-compatible task, but deciding which sample should be placed under the microscope is not robot compatible.

It is hard to put a metric on the robotic compatibility factor. Instead, we showed our list of tasks to a group of robotics experts, and asked them to give us their opinions on which tasks are, or are not, robot-compatible. It is important to note that robot-compatibility can be built into a design, if you plan it that way ahead of time. So for many of the planetary surface tasks, we can only say that the task could be robot-compatible, if the designers decide to make it so.

\section{Task criticality}

What are the consequences of a failure of the robot to accomplish the task? If it is just a minor inconvenience, then you do not have to design in as much redundancy as you would if the failure would lead to a catastrophe.

\section{$3.2 b$ Benefit aspects}

\section{Task duration}

How much spacewalking or surface excursion time might be saved if a robot could do the task?

\section{Task frequency}

How often would that block of time be saved? If the task requires five hours, but only happens once during the whole mission, then maybe that is not the most beneficial way to use robots. Instead perhaps, we should pay attention to tasks that require an hour every day.

\section{Task pervasiveness}

If a task is performed at lots of different places, it probably is more beneficial to roboticize it.

\section{Human factors}

Something that the crew would consider as challenging, interesting and fun would not be as beneficial to roboticize as something that was tedious, boring, strenuous, or dangerous.

\section{Distance from safety}

Finally, how far away from the airlock is the human going to be if they perform this task? If they are very close to the airlock, then they spent less time getting to the work site, and it is quicker to get back to the airlock in an emergency. All other things being equal, it would be more beneficial to roboticize tasks that take place farther from the airlock.

For planetary surface missions, we identified a larger number of tasks that would be associated with surface excursions, because surface traverses by humans would be a primary science activity. 


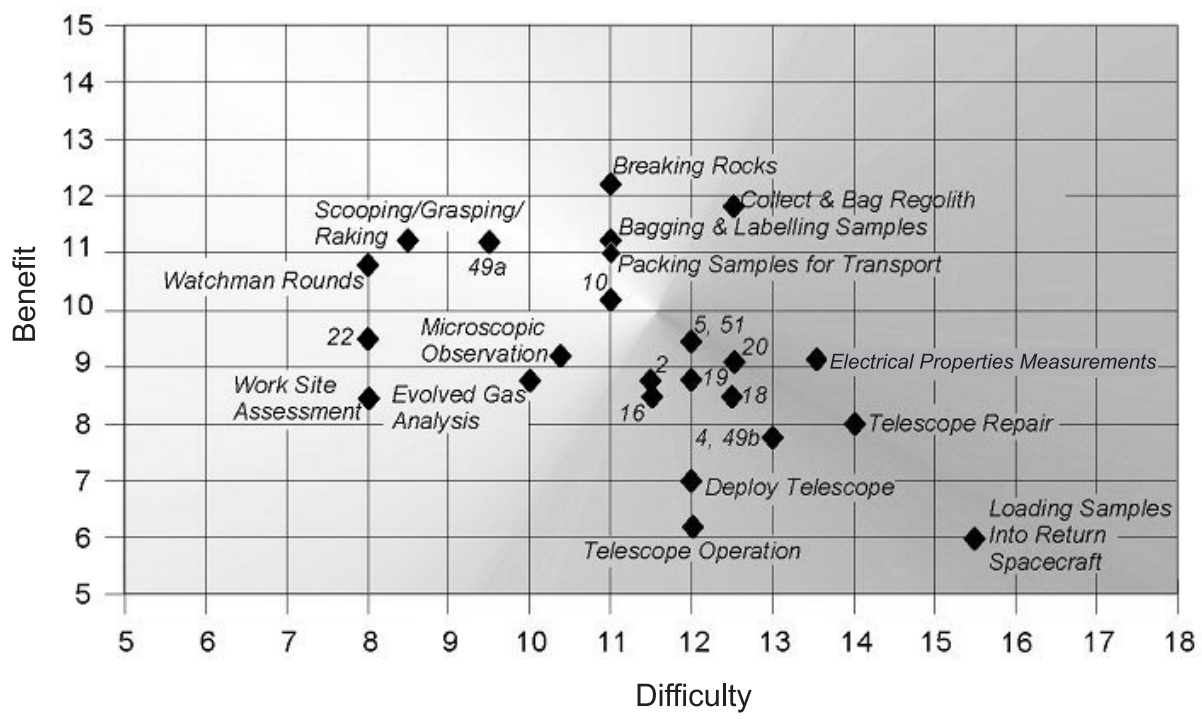

Figure 1. Payback for robotic tasks that occur in conjunction with human activity. Lower 'difficulty' numbers and higher 'benefit' numbers represent the most useful robotic tasks for early development. Complete list of tasks (represented here by numbers only) may be found in Cooper and O'Donnel (1999).

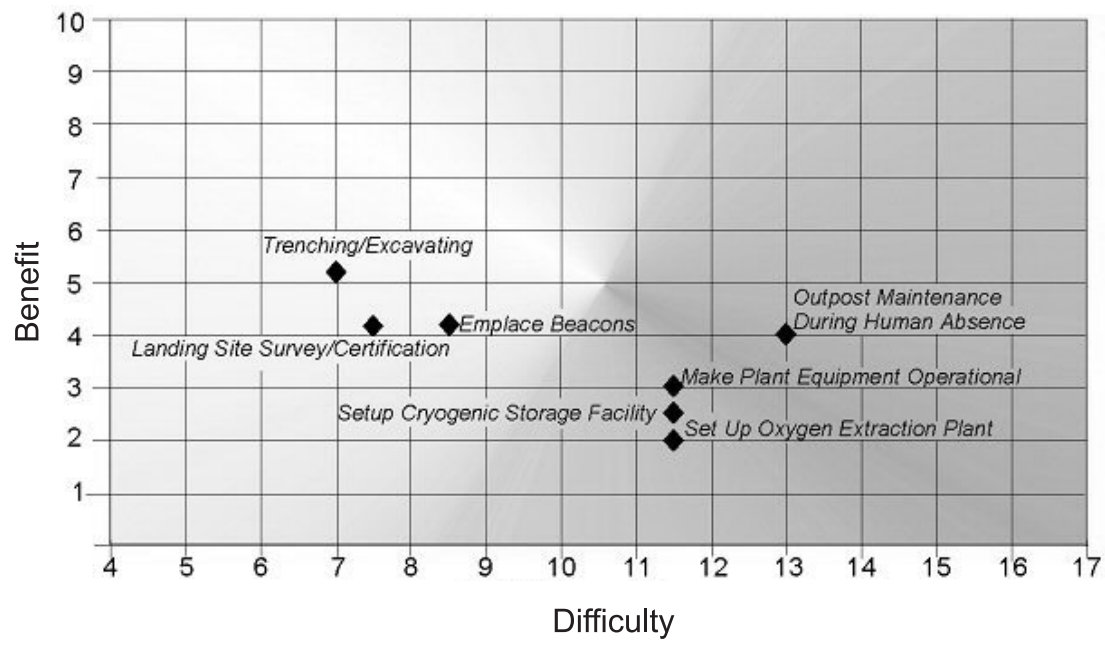

Figure 2. Payback for long-term enabling technology tasks. Robot development should focus on the low-difficulty, highpayback tasks in the upper left quadrant.

\subsection{Results}

The highest-payback tasks for the 'Robot Assistant' are shown in the upper left quadrant of figure 1 - least difficulty, greatest benefit. For example, scooping, grasping and raking scores well as a task that would give a high payback for robotics development. Breaking rocks, observing them in the field with a microscope, and autonomous surface traverses also got high marks. By comparison, loading samples into a return spacecraft was not very useful.

Enabling technology for planetary surface mission phases includes some rather daunting tasks, and, as we mentioned before, the question to be asked is not whether it makes sense for a robot to do these things instead of a human. There are no humans in the scenario. So how do we score them with respect to benefit? It may be useful to ask ourselves a slightly different question: Which tasks should be roboticized, which tasks should be developed for hard-automation, and which tasks should not be considered for now?

Because these are enabling technologies for future human missions to the Moon, we do have to consider them, but it is nevertheless useful to know that trenching and excavating is a higherpayoff task than landing site survey and certification. From the standpoint of efficient allocation of resources, the high-benefit tasks should be 
addressed first. Technologies developed and lessons learned can then be applied to the more difficult, or possibly less beneficial, tasks (figure 2).

\section{3a Pervasive subtasks}

\section{Closeout images}

There was a third category of tasks that we call 'pervasive subtasks' (figure 3) that have to occur in just about any robotic mission. For example, when a repair is completed, it is very important to take some pictures of the completed job. These are typically referred to as 'close-out' images. They allow you to take a careful look at your work after you get back inside, and then, in a leisurely fashion, you can decide if the job was done well enough, or if instead you need to plan another spacewalk to go out and fix it again.

\section{Situational awareness}

Another important 'pervasive subtask' is situational awareness. An astronaut working in a spacesuit has limited vision and limited range of motion, and it is very easy to bump into or get snagged on something. That is one of the reasons that at least two people should go out on each spacewalk - to have a buddy there in case of trouble. So, having a robot that can just watch out for problems (being managed perhaps by a human who is just inside) can be a very useful option.

\section{Imagery}

We have seen that the capability of taking still or live imagery is important in many of the highpayback tasks. This makes sense, if you consider the fact that you need to be able to see what you are doing, whether you are a robot or a human. And if you are a robot, you probably need visual information to a greater extent, because you lack the sense of touch feedback that a human has.

When astronauts are performing maintenance during a spacewalk, having a camera on the end of a manipulator would allow them to peek around corners or under things. A flying eyeball for inspection of the exterior surface of the spacecraft helps identify problems, assess work sites, find leaks, and in general allows better planning before the spacewalk begins. On a planetary surface, having a camera on the end of a manipulator might allow the crew to choose samples for inspection, without having to pick them all up, just to throw most of them back down.

This imagery capability is also central to landing site survey and qualification - it is useful to see what is there in detail so that you can plan ahead for challenges, or you may decide to pick another location for your initial base camp. These tasks involve mobility and the ability to capture images. Since they do not involve manipulating objects, they are generally the easiest things to do with robots.

\section{Mobility and manipulation}

Some tasks involve manipulation in addition to mobility. Among these, the highest-payback tasks involve maintenance. When we begin designing the systems that will allow humans to live on the Moon, we should remember to add robotic interfaces to things that could break, and to include robotic compatibility in the design. Things that are robotically compatible should be humancompatible as well, because that adds redundancy to the design.

\section{Lessons learned}

We need to plan ahead intelligently to use robots as efficiently as possible. We have learned a few lessons from international space station and from terrestrial robotics applications, about what works and what does not work so well. Here is our short list of those lessons learned.

- Successful work systems rarely double as technology demonstrations. Usually, the prototype is a way to learn how to do things better, and we do not expect that prototype to stay on the job for years. We need to understand the robotic system and have some operational experience with it, then develop the 'workhorse' based on that experience.

- Design tasks for single-arm operations. In space, you need to hold on to whatever you are working on. On a low-gravity planet, you may need to hold onto something to keep your balance. The robot will probably require the same thing, and the object is to make the task operable by either human or robot.

- Minimize fasteners and actuators. Find a way to use only two bolts instead of twenty. Only one switch instead of ten, and so forth.

- Provide clear visual and physical access and visual cues and design those visual cues with the imaging system in mind. In other words, do not make an orange-and-white target for a monochrome (black-and-white) visual system.

- Minimize motion requirements. If you need to turn a valve, build a tool with a motor in it, instead of a steering wheel or lever.

- Provide alignment guides. Make sure that the alignment guides are located where the vision system can see them.

- Allow for proper tolerances between parts - there is a balance between too tight a tolerance and 


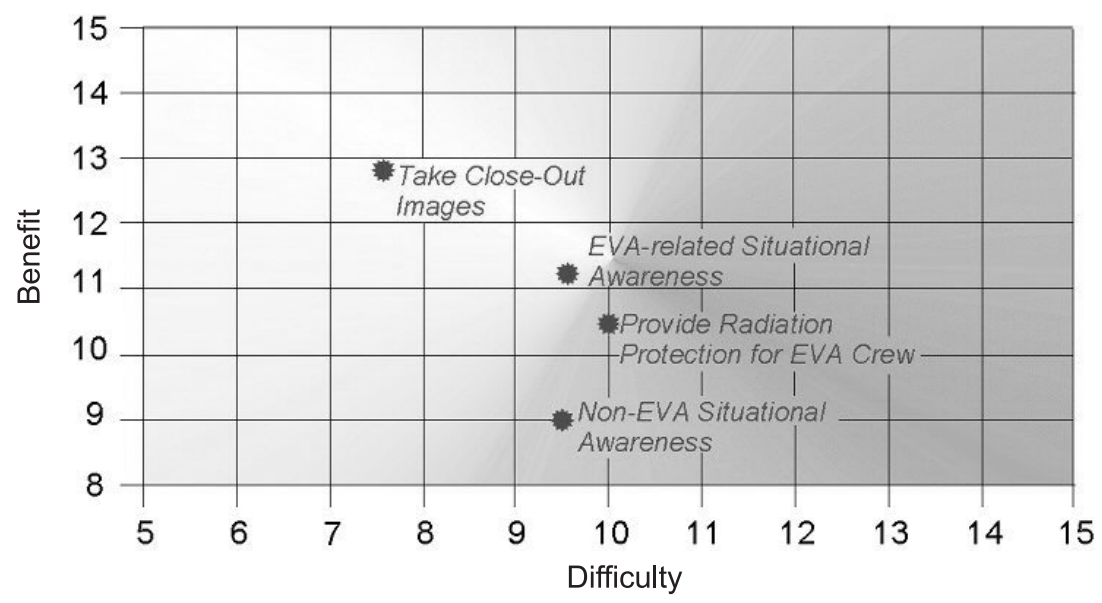

Figure 3. Pervasive subtasks, activities and capabilities. These items are candidates for automated subroutines (other candidates are discussed in section 2.1.2).

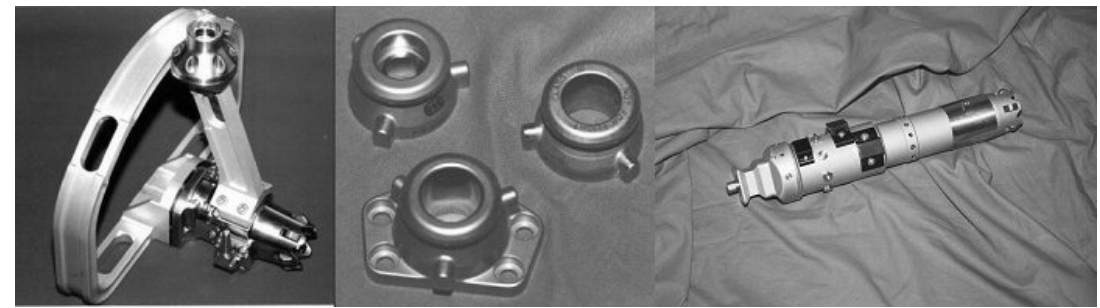

Figure 4. The microconical tool, developed by Oceaneering Space Systems in support of the International Space Station (ISS).

too loose a tolerance, and either one can cause problems.

- Provide status indicators for robot-activated functions. Robots usually have no tactile feedback system, so they need some way of knowing when they have made the connection, tightened the bolt enough, and so on.

- Provide clear identification of objects and directions. UP and DOWN do not apply if you do not have a neurovestibular system.

- Provide operational margins - design for the mid range of capabilities. You may expect a mechanism to work continuously for fifteen years, but you should make it easy to replace anyway.

- Limit the amount of force required to operate mechanisms. That makes it more humancompatible, and more robot-compatible at the same time.

\subsection{Recommendations}

\section{4 a RoboTractor}

The development of a robotic excavation/soilmoving capability is a critical component of an overall robotic exploration system.

\section{$3.4 b$ Engineering standards}

Equipment for lunar development should be designed with open-ended operations in mind no more 'missions' that have a defined end-point. Design consideration must be given to commonality standards so that every piece of equipment sent to the Moon now and in the future can be interfaced (added to or cannibalized/reconfigured) with all other equipment.

\section{4c Incorporate a telerobotic control mode}

Incorporate a limited tele-operation control mode (where feasible) to robotic systems to permit them to be 'driven out' of situations where supervisory or autonomous control technologies may not be adequate.

\section{4d Design early for robots}

Make provisions to include robot capabilities as part of the overall planetary work system early in the planning stages of future missions. Incorporate robot-specific interfaces so that tasks can migrate to robotics as the capabilities are fielded. Figure 4 shows a device called the micro-conical tool. It is 
used on the Space Station as an attachment device. It was designed to be operated either by a human or a robot - even though we do not have robots on Space Station yet that can actually use this. When a human uses it, the 'steering-wheel' device on the left is hooked up to the microconical, and the human can easily turn it. When a robot is working, the robot uses the rotating tool shown on the right.

\section{4e Develop robot surrogate capabilities}

Continue to develop robot surrogate-type capabilities. These options provide a unique capability to flexibly respond to contingency and emergency situations when suited human crew members are not available.

\section{$3.4 f$ Plan ahead}

Identify overhead tasks associated with the primary job so that time lines accurately reflect the required work. When a more accurate timeline is established the need for robot capabilities and interfaces becomes apparent. When the need is defined, a more efficient system (combining human and robotic tasks) can be developed within a context that best supports the overall mission.

Historically, a lack of understanding of what kind of work robots could actually perform in space led to a reluctance to place them in the critical path of assembly or maintenance activities. Therefore, robotic accommodations were not widely incorporated into the architecture due to cost, weight, volume, etc. As development continued and new tasks were discovered, it was difficult and even more expensive to retrofit the space hardware for robotic operations.

\section{$3.4 \mathrm{~g}$ Build a little, test a little}

Provide robot demonstrations early in the planning stages of a mission to instill confidence and to showcase capabilities to mission planners and managers. Robot accommodations and tasking will occur when planners and managers understand what robots can do and what is required (accommodations) for them to perform the tasks.

\section{4h Mobility is key}

Mobility is an enabling robot capability. Almost all tasks examined for robots include a requirement for this capability. Technologies that implement or facilitate robot mobility are critical to future planetary exploration.

\section{Conclusions}

Telerobotic technologies are beneficial to future lunar development, and should be incrementally developed by starting with those tasks which are the most beneficial and the least difficult to roboticize. When these technologies are proven (albeit at the microscopic/microwatt level), they will signal the beginning of the true 'space age' - large-scale space operations comprised of a mix of human, teleoperated, and autonomous robotic participants.

\section{Acknowledgements}

This work was funded in part by NASA Johnson Space Center and Oceaneering Space Systems, Houston.

\section{References}

Cooper B L and O'Donnel J 1999 Robot Associate Study, unpublished white paper presented to NASA Johnson Space Center Automation, Robotics, and Simulation Division, pp. 1-89.

Sharpe B and Schrunk D 2002 Malapert Mountain: Gateway to the Moon; Presented at the World Space Congress, CoSpar Session, Houston, Texas.

Sharpe B, Schrunk D and Thangavelu M 2003 Lunar Reference Mission: Malapert Station; Proceedings of the International Lunar Conference 2003 (ILEWG-5), Kohala Coast, Hawaii, pp. 259-264, 16-22 November. 